\title{
Electron exchange model potential: Application to positronium-helium scattering
}

\author{
P. K. Biswas and Sadhan K. Adhikari \\ Instituto de Física Teórica, Universidade Estadual Paulista, 01 405-900 São Paulo, São Paulo, Brazil \\ (Received 17 July 1998; revised manuscript received 8 September 1998)
}

\begin{abstract}
The formulation of a suitable nonlocal model potential for electron exchange is presented, checked with electron-hydrogen and electron-helium scattering, and applied to the study of elastic and inelastic scattering and ionization of orthopositronium (Ps) by helium. The elastic scattering and the $n=2$ excitations of Ps are investigated using a three-Ps-state close-coupling approximation. The higher $(n \geqslant 3)$ excitations and ionization of Ps atoms are treated in the framework of the Born approximation with present exchange. Calculations are reported of phase shifts and elastic, Ps excitation, and total cross sections. The present target elastic total cross section agrees well with experimental results at thermal to medium energies. [S1050-2947(99)04201-8]

PACS number(s): 34.10.+x, 36.10.Dr
\end{abstract}

\section{INTRODUCTION}

The neutral Ps beam provides a great deal of advantage over charged projectiles as a probe to study the structure of atoms, molecules, and surface. Recently, there has been a great deal of interest in positronium- (Ps-) atom scattering due to the improvement of Ps sources and Ps beams. Total scattering cross sections of ortho-Ps, which has a larger lifetime than para Ps, have been measured for various targets $[1,2]$ with an objective of understanding the Ps-interaction dynamics with matter. Among all Ps-atom systems, the positronium-hydrogen (Ps-H) system is the simplest and is of special theoretical interest [3]. However, due to experimental difficulties in obtaining a nascent-hydrogen atomic target, there has been no experimental study of Ps-H scattering. The next most complicated Ps-atom system is the positroniumhelium (Ps-He) system in which there are good experiments on total cross sections $[1,2]$. However, there are no theoretical studies [4-7] which can account for the measured total cross sections of ortho-Ps-He scattering. We address the present study towards an understanding of the measured total cross sections of Ps-He scattering at low and medium energies using a suitably developed model exchange potential.

The interaction of a neutral Ps atom with a neutral atom or molecule is very much different from that of charged electrons and positrons with neutral targets [8]. In any Ps-atom scattering, the elastic and even-parity state transition direct amplitudes to the close-coupling approximation (CCA) are zero [3] due to internal charge and mass symmetry of Ps. In addition, the adiabatic polarization potential is also zero and the electron-exchange mechanism appears as the main driving force at low energies apart from the correction expected from polarization and the Van der Waals force [5]. This was not the case for electron-impact scattering, where both the direct and exchange interactions play a role in determining the solution of the scattering equations. The Ps-atom system allows the possibility for studying the effect of exchange in an environment characteristically different from that of the electron-atom system due to the composite nature and the underlying charge and mass symmetry of Ps. Recently, in addition to the total cross section at medium to high energies [1], thermalization of Ps in gaseous He has also been mea- sured [2]. However, it is of serious concern that dynamical calculations with the reliable and widely used staticexchange model with usual antisymmetrization [4-6] fail severely yielding very large total cross sections compared to the measured data $[1,2]$, especially at low energies. The experiments of Refs. [1,2] are consistent among themselves. They collectively suggest a lowering trend of cross sections from a peak at $20 \mathrm{eV}$ towards lower energies. This trend is missing from all previous published calculations. Moreover, due to the large error bar on the measured cross section at 10 $\mathrm{eV}$ of Ref. [1] and the absence of data near Ps excitation and ionization thresholds, it is not clear whether the cross section has a minimum or not in this energy region. The present study also addresses this feature from a theoretical point of view.

The proper inclusion of the exchange effect is a major technical obstacle in performing dynamical calculations in complex systems [9]. The effect of electron exchange is usually accounted for in a quantum dynamical calculation through the antisymmerization of the wave function, which introduces nonorthogonal functions to these calculational schemes including the usual static-exchange model. These antisymmetrization schemes with nonorthogonality defects lead to overcompleteness in the Hilbert space and associated theoretical and numerical difficulties in the CCA and related formalisms. Moreover, when short-range (exchange) correlations are important, the CCA converges very slowly [10]. Several discussions and prescriptions to remedy this defect have appeared in the literature in connection with electron impact scattering [9]. This problem has been overcome to some extent in electron-impact scattering using different methodologies - with an essentially exact (variational) treatment of exchange in simpler cases, with effective correlation and suitable model potentials [11] for larger targets. Gross deviations of previous calculations [4-6] on Ps-He scattering from measurements at low energies [2] could be a consequence of the nonorthogonality defect and/or the inadequacy of the correlation effect in exchange-dominated Ps-impact scattering, especially at low energies.

To address this problem, we choose to remove the nonorthogonality from the exchange kernel of the momentumspace CCA equation by using a suitable model potential. The 
additional simplicity of the present exchange potential makes it very attractive for performing dynamical calculations in many-electron systems. The exchange model is shown to be readily applicable to electron- and Ps-impact scattering problems. In order to test the generality and reliability of the exchange model, we apply it to electron-hydrogen $\left(e^{-}-\mathrm{H}\right)$ and electron-helium $\left(e^{-}-\mathrm{He}\right)$ scattering, in addition to Ps-He scattering.

We present a theoretical study of ortho-Ps-He scattering employing a three-Ps-state CCA scheme in momentum space where the usual nonorthogonal exchange kernel arising from antisymmetrization is replaced by the present model exchange potentials. The helium atom is always assumed to be in its initial ground state and the $\operatorname{Ps}(1 s), \operatorname{Ps}(2 s)$, and $\operatorname{Ps}(2 p)$ states are included in the coupled-channel calculation. Being the lightest atom, Ps is more vulnerable to excitation than the inert helium atom in Ps-He scattering. Also, the Ps-excitation thresholds are the lowest ones in this system. Hence, the present three-Ps-state model seems to be a reasonable one to describe Ps-He scattering from low to medium energies. The cross sections for higher discrete and continuum excitations of Ps atoms are calculated in the framework of the first Born approximation including present exchange. These Born cross sections are added to the above three-Ps-state cross sections to predict the target elastic total cross section.

The plan of the paper is as follows. In Sec. II we present the model exchange potential for electron-impact scattering and numerical results for electron scattering by $\mathrm{H}$ and $\mathrm{He}$. In Sec. III we present the model exchange potential for Psimpact scattering and numerical results for Ps scattering by He. Finally, in Sec. IV we present a summary of our findings.

\section{EXCHANGE POTENTIAL FOR ELECTRON-IMPACT SCATTERING}

Although we are mostly interested in developing an exchange potential for Ps-impact scattering in this work, first we illustrate and check our model in the case of electronatom scattering where the exchange potential is well under control. We develop the present exchange model for $e^{-}-\mathrm{H}$ elastic scattering using a $\mathrm{H}(1 s)$ orbital and finally extend it to the case of inelastic scattering by a complex target described by a Hartree-Fock (HF) wave function. The exchange potentials are derived from the following nonorthogonal exchange transition amplitude [11]:

$$
\begin{aligned}
g\left(\mathbf{k}_{f}, \mathbf{k}_{i}\right)= & -\frac{1}{2 \pi} \int d \mathbf{r}_{1} d \mathbf{r}_{2} \phi^{*}\left(\mathbf{r}_{2}\right) \frac{1}{r_{12}} \phi\left(\mathbf{r}_{1}\right) \\
& \times \exp \left[i\left(\mathbf{k}_{i} \cdot \mathbf{r}_{2}-\mathbf{k}_{f} \cdot \mathbf{r}_{1}\right)\right]
\end{aligned}
$$

where the position vector of the incident (target) electron is $\mathbf{r}_{2}\left(\mathbf{r}_{1}\right)$. Here $\phi$ is the wave function of $\mathrm{H}, \mathbf{k}_{i}\left(\mathbf{k}_{f}\right)$ is the initial (final) momentum of the incident electron, and $\mathbf{r}_{12}=\mathbf{r}_{1}-\mathbf{r}_{2}$. Amplitude (2.1) is the leading term of the exchange amplitude at large energies [12] and also the usual starting point for deriving model exchange potentials [11]. To remove the nonorthogonality aspect, we seek an exchange potential of the form

$$
g\left(\mathbf{k}_{f}, \mathbf{k}_{i}\right) \sim \int d \mathbf{r} \phi^{*}(\mathbf{r}) U\left(\mathbf{r}, \mathbf{k}_{i}, \mathbf{k}_{f}\right) \phi(\mathbf{r})
$$

where the form of $U$ is to be determined. We consider the integration over the coordinate of the final projectile electron $\mathbf{r}_{1}$ of Eq. (2.1) below. Using $\phi(\mathbf{r})=\pi^{-1 / 2} \alpha^{3 / 2} \exp (-\alpha r)$, taking Fourier transformation, and performing the integration over $\mathbf{r}_{1}$, we obtain

$$
\begin{aligned}
I & \equiv \int d \mathbf{r}_{1} \frac{1}{r_{12}} \phi\left(\mathbf{r}_{1}\right) \exp \left(-i \mathbf{k}_{f} \cdot \mathbf{r}_{1}\right) \\
& =\frac{4 \alpha^{5 / 2}}{\pi^{3 / 2}} \int d \mathbf{q} \frac{\exp \left(-i \mathbf{k}_{f} \cdot \mathbf{r}_{2}\right)}{\left(\mathbf{k}_{f}-\mathbf{q}\right)^{2}} \frac{\exp \left(i \mathbf{q} \cdot \mathbf{r}_{2}\right)}{\left(q^{2}+\alpha^{2}\right)^{2}}
\end{aligned}
$$

Any average value prescription for $\left(\mathbf{k}_{f}-\mathbf{q}\right)^{2}$ in Eq. (2.3) will reduce Eq. (2.1) to form (2.2). Then, in the model exchange potential, the final- and initial-state wave functions will be expressed in terms of the same coordinates. Recalling that the internal kinetic energy of $\mathrm{H}\left(q^{2} / 2\right)$ is given by $\alpha^{2} / 2$ in atomic units, we take the average of $q^{2}$ as $\alpha^{2}$, and set $\left(\mathbf{k}_{f}\right.$ $-\mathbf{q})^{2} \approx\left(k_{f}^{2}+\alpha^{2}\right)$, where the average value of the scalar product is assumed to be zero. After taking an inverse Fourier transformation in Eq. (2.3), the final model exchange potential takes the following simple form:

$$
g\left(\mathbf{k}_{f}, \mathbf{k}_{i}\right) \approx \frac{-2}{k_{f}^{2}+\alpha^{2}} \int \phi^{*}\left(\mathbf{r}_{2}\right) \exp \left(i \mathbf{Q} \cdot \mathbf{r}_{2}\right) \phi\left(\mathbf{r}_{2}\right) d \mathbf{r}_{2},
$$

where $\mathbf{Q}=\mathbf{k}_{i}-\mathbf{k}_{f}$. Although we derived Eq. (2.4) for elastic scattering, this result is straightforwardly extendable to inelastic $e^{-}-\mathrm{H}$ scattering to a final $\mathrm{H}(2 s, 2 p), \mathrm{H}(3 s, 3 p, 3 d)$, etc. orbital. In such cases the final model exchange potential for the transition from state $\nu$ to $\nu^{\prime}$ becomes

$$
g_{\nu^{\prime} \nu}\left(\mathbf{k}_{f}, \mathbf{k}_{i}\right) \approx-\frac{2}{k_{f}^{2}+\alpha_{\nu}^{2}} \int \phi_{\nu^{\prime}}^{*}\left(\mathbf{r}_{2}\right) \exp \left(i \mathbf{Q} \cdot \mathbf{r}_{2}\right) \phi_{\nu}\left(\mathbf{r}_{2}\right) d \mathbf{r}_{2},
$$

where the parameter $\alpha_{\nu}$ refers to the initial state $\nu$. The form of this potential is not time-reversal symmetric; for a symmetric form, see the discussion after Eq. (2.8).

Similar model potentials were derived by Ochkur and also by Rudge [12]. Ochkur's result is obtained by setting $\alpha=0$ in the prefactor of Eq. (2.5). Rudge's result corresponds to taking the prefactor $\left(k_{f}^{2}+\alpha^{2}\right)^{-1}=\left(k_{f}-i \alpha\right)^{-2}$. The model exchange potential (2.5) has the following desirable physical properties. This potential is the strongest at the lowest possible energy $\left(k_{f}=0\right)$ for the weakest bound atomic orbital $\left(\alpha_{\nu} \rightarrow 0\right)$. Hence, the effect of exchange is more pronounced at low energies for the loosely bound orbitals.

For a general $\mathrm{HF}$ wave function, $\psi\left(\mathbf{r}_{1}, \ldots, \mathbf{r}_{j}, \ldots, \mathbf{r}_{N}\right)$ $=\mathcal{A}\left[\Pi_{j=1}^{N} \phi_{j}\left(\mathbf{r}_{j}\right)\right]$, where $\mathcal{A}$ is the antisymmetrization operator and the position vectors of the electrons are $\mathbf{r}_{j}, j$ $=1,2, \ldots, N$, and the atomic orbitals $\phi_{j}(\mathbf{r})$ have the following form: 


$$
\phi_{j}(\mathbf{r}) \equiv \sum_{\kappa} a_{\kappa j} \phi_{\kappa j}(\mathbf{r})
$$

Summing over appropriate target electrons $j$ and allowing for inelastic channels, the full exchange potential is given by

$$
\begin{aligned}
B_{E, \nu^{\prime} \nu}\left(\mathbf{k}_{f}, \mathbf{k}_{i}\right)= & \sum_{j} s_{j} g_{j}=-\sum_{j} \sum_{\kappa \kappa^{\prime}} s_{j} \frac{2 a_{\kappa j} a_{\kappa^{\prime} j}}{D_{\kappa \kappa^{\prime} j}} \\
& \times \int \phi_{\kappa^{\prime} j}^{*}(\mathbf{r}) \exp (i \mathbf{Q} \cdot \mathbf{r}) \phi_{\kappa j}(\mathbf{r}) d \mathbf{r},
\end{aligned}
$$

with

$$
D_{\kappa \kappa^{\prime} j}=\left[k_{f}^{2}+\alpha_{\kappa j}^{2}\right]
$$

where $\phi_{\kappa j}(\mathbf{r})$ is the $\kappa$ th function of the $j$ th electron and $\alpha_{\kappa j}$ refers to the initial state and $s_{j}(=0, \pm 1)$ has to be chosen according to the spin symmetry.

The model potential (2.7) with prefactor $D_{\kappa \kappa^{\prime} j}$ of Eq. (2.8) is not time-reversal symmetric. However, if we perform the integration over the initial projectile electron $\mathbf{r}_{2}$ in Eq. (2.1) first, and carry on a similar procedure, we obtain exchange potential (2.7) with $D_{\kappa \kappa^{\prime} j}=\left(k_{i}^{2}+\alpha_{\kappa^{\prime} j}^{2}\right)$, where $\alpha_{\kappa^{\prime} j}$ refers to the final state. These two possibilities suggest the following symmetric prefactor:

$$
D_{\kappa \kappa^{\prime} j}=\left[\left(k_{i}^{2}+k_{f}^{2}\right) / 2+\left(\alpha_{\kappa j}^{2}+\alpha_{\kappa^{\prime} j}^{2}\right) / 2\right]
$$

in Eq. (2.7). The two possibilities (2.8) and (2.9) correspond to two averages. At high energies, the model exchange potential (2.7) with different averaging prescriptions leads to the Oppenheimer exchange potential [13]. However, at low energies the cross section is sensitive to the averaging procedure and the value of the parameter $\alpha$ in prefactors (2.8) or (2.9). This sensitivity may well be exploited to tune the parameter $\alpha$ of a particular averaging procedure in order to obtain a better fit with experiment at low energies.

Although the model potential (2.7) is derived for the ground state of the atomic target, it is straightforward to see that the same result is also valid for target excitations in the final state using a similar averaging prescription. Hence model potential (2.7) is equally valid for both elastic and inelastic scattering by the target.

We have used the exchange potential (2.7) in $e^{-}-\mathrm{H}$ and $e^{-}$-He scattering and calculated the elastic cross sections. We also demonstrate the effect of different averaging procedures-symmetric and nonsymmetric - and the variation of the parameter $\alpha$ whenever relevant. In the case of $e^{-}-\mathrm{H}$ scattering, we exhibit the results for an elastic cross section in a coupled $\mathrm{H}(1 s, 2 s, 2 p)$ model using the above exchange potential in the symmetric form (2.9) with the exact value of the parameter $\alpha$. For $e^{-}$-He scattering we present results for an elastic cross section in the staticexchange model using the symmetric form (2.9). In the case of He we present a variation of the parameter so as to obtain a better fit with experiment.

In Fig. 1 we present results for $e^{-}$-H scattering using Eqs.

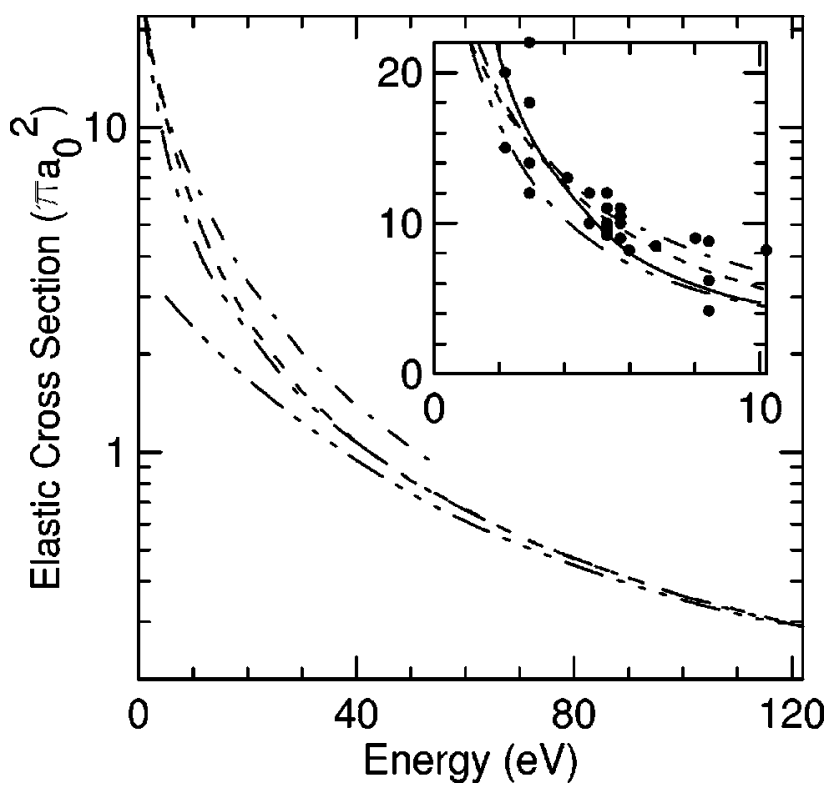

FIG. 1. Elastic electron-hydrogen cross section: present static exchange model (dashed-double-dotted line); present Born (dashed line); present $\mathrm{H}(1 s, 2 s, 2 p)$ model (full line); total first Born with Oppenheimer exchange (dashed-triple-dotted line); polarized orbital model by Temkin and Lamkin [10] at lower energies $(<10 \mathrm{eV})$ and CCA model by Callaway [15] at higher energies (dashed-dotted line); experiment (solid circles, Ref. [14]) $[\mathrm{H}(1 s, 2 s, 2 p) \mathrm{CCA}$ model results by Burke and Schey [10] are very close to Temkin and Lamkin and are not shown].

(2.7) and (2.9), where we exhibit the exchange Born, staticexchange, and $\mathrm{H}(1 s, 2 s, 2 p)$ cross sections without variation of the parameter $\alpha$. In this figure we compare the low-energy cross sections with experimental results [14] and the calculations by Temkin and Lamkin [10]. At medium energies the results are compared with an essentially converged calculation of Callaway [15]. We also plot the total first Born cross section with Oppenheimer exchange [13]. At low energies the present $\mathrm{H}(1 s, 2 s, 2 p)$ cross sections are an improvement over the present static-exchange cross sections. At higher energies they are essentially identical and only the staticexchange results are shown. At large energies, as expected, the present cross sections tend to the total first exchangeBorn (Born+Oppenheimer exchange) results. Both at low and medium energies the agreement of the present cross sections with the results of other workers is encouraging. We verified that both the exchange Born and static-exchange cross sections are sensitive to the variation of the parameter $\alpha$ in the prefactor (2.9). We demonstrate the effect of such variation at low energies in the study of $e^{-}$-He scattering where it seems more relevant.

In Fig. 2 we plot the present static-exchange cross section of electron-helium scattering for the model exchange potential given by Eqs. (2.7) and (2.9) with the HF helium wave function of Ref. [16]. In this case we present results for first exchange-Born and static-exchange elastic cross sections with the exact parameters $\alpha$ 's. Here we also present results for static-exchange cross sections with modified value for the parameters $\alpha\left[\left\langle q^{2}\right\rangle=(0.4 \alpha)^{2}\right]$ in the prefactor (2.9) for both $\kappa$ and $\kappa^{\prime}$ corresponding to initial and final states, respectively. [The parameters in the helium wave function under 


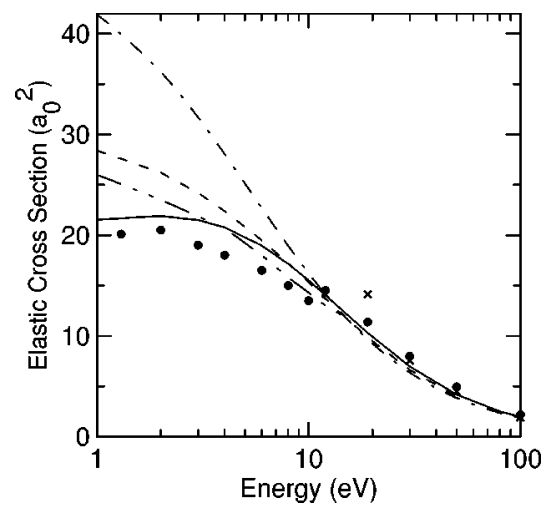

FIG. 2. Elastic electron-helium cross section: present static exchange with exact parameters $\alpha$ (dashed-dotted line); present Born with exact $\alpha$ (dashed line); present static exchange with modified $\alpha$ (full line); $\mathrm{He}\left(1 s, 2{ }^{1} s, 2{ }^{3} s, 2{ }^{1} p, 2{ }^{3} p\right)$ CCA calculation of Burke et al. (dashed-double-dotted line, Ref. [18]); experiment (solid circles and crosses, Ref. [17]).

the integral in Eq. (2.7) are left unchanged, as they should be.] The results are compared with experimental results and the five-state $\left[\mathrm{He}\left(1 s, 2{ }^{1} s, 2{ }^{3} s, 2{ }^{1} p, 2{ }^{3} p\right)\right]$ CCA calculation of Burke et al. [18] using the model exchange potential.

The present static-exchange cross sections agree reasonably with experiment [17] at medium to high energies. The variation of the parameter $\alpha$ in this case has led to good agreement with experiment and the CCA calculation of Burke et al. [18] at lower energies. For obtaining a better agreement with experiment, the effect of excitation and polarization of the target should be taken into account. This could be done by considering a coupled-channel calculation with helium excitations as in the electron-hydrogen scattering considered above. With this reliability achieved in the $e^{-}-\mathrm{H}$ and $e^{-}$-He systems, we extend this exchange model to Ps impact cases.

\section{EXCHANGE POTENTIAL FOR POSITRONIUM-IMPACT SCATTERING}

\section{A. Formulation}

Here, we first develop the present exchange model potential for Ps-H elastic scattering using a $\mathrm{H}(1 s)$ orbital and finally extend it to inelastic Ps scattering by a many-body target described by a HF wave function. We start with the following nonorthogonal exchange transition amplitude [11]:

$$
\begin{aligned}
g\left(\mathbf{k}_{f}, \mathbf{k}_{i}\right)= & -\frac{1}{\pi} \int d \mathbf{x} d \mathbf{r}_{1} d \mathbf{r}_{2} \phi^{*}\left(\mathbf{r}_{2}\right) \chi^{*}\left(\mathbf{t}_{1}\right) \frac{1}{r_{12}} \phi\left(\mathbf{r}_{1}\right) \\
& \times \chi\left(\mathbf{t}_{2}\right) \exp \left[i\left(\mathbf{k}_{i} \cdot \mathbf{s}_{2}-\mathbf{k}_{f} \cdot \mathbf{s}_{1}\right)\right]
\end{aligned}
$$

where the position vector of the electron (positron) of Ps is $\mathbf{r}_{2}(\mathbf{x})$. Here $\mathbf{s}_{j}=\left(\mathbf{x}+\mathbf{r}_{j}\right) / 2, \mathbf{t}_{j}=\left(\mathbf{x}-\mathbf{r}_{j}\right), j=1,2$, and $\chi(\phi)$ is the wave function of Ps $(\mathbf{H})$. As in the preceding section, to remove the nonorthogonality defect we seek an exchange potential of the form

$$
g\left(\mathbf{k}_{f}, \mathbf{k}_{i}\right) \sim \int d \mathbf{r} d \mathbf{t} \phi^{*}(\mathbf{r}) \chi^{*}(\mathbf{t}) U\left(\mathbf{r}, \mathbf{t}, \mathbf{k}_{i}, \mathbf{k}_{f}\right) \phi(\mathbf{r}) \chi(\mathbf{t})
$$

where $U$ is to be determined. We consider the integration over the coordinate of the final projectile electron $\mathbf{r}_{1}$ of Eq. (3.1) below. Using $\phi(\mathbf{r})=\pi^{-1 / 2} \alpha^{3 / 2} \exp (-\alpha r)$ and $\chi(\mathbf{t})$ $=\pi^{-1 / 2} \beta^{3 / 2} \exp (-\beta t)$, taking Fourier transformation, the integral $\mathcal{I}$ over $\mathbf{r}_{1}$ is given by

$$
\begin{aligned}
\mathcal{I} \equiv & \int d \mathbf{r}_{1} \chi^{*}\left(\mathbf{t}_{1}\right) \frac{1}{r_{12}} \phi\left(\mathbf{r}_{1}\right) \exp \left(-i \mathbf{k}_{f} \cdot \mathbf{s}_{1}\right) \\
= & \frac{4(\alpha \beta)^{5 / 2}}{\pi^{4}} \int d \mathbf{p} d \mathbf{q} \frac{\exp \left(-i \mathbf{k}_{f} \cdot \mathbf{r}_{2} / 2\right)}{\left(\mathbf{k}_{f} / 2-\mathbf{p}+\mathbf{q}\right)^{2}} \\
& \times \frac{\exp \left(i \mathbf{q} \cdot \mathbf{t}_{2}\right)}{\left(q^{2}+\beta^{2}\right)^{2}} \frac{\exp \left(i \mathbf{p} \cdot \mathbf{r}_{2}\right)}{\left(p^{2}+\alpha^{2}\right)^{2}}
\end{aligned}
$$

Again we employ an average value prescription for $\left(\mathbf{k}_{f} / 2\right.$ $-\mathbf{p}+\mathbf{q})^{2}$ in Eq. (3.3) which will reduce Eq. (3.1) to form (3.2). Recalling that the internal kinetic energies of $\mathrm{H}$ (represented by $\left.p^{2} / 2 m ; m=1\right)$ and Ps $\left(q^{2} / 2 m ; m=1 / 2\right)$ are given by $\alpha^{2} / 2$ and $\beta^{2}$ in atomic units, we take the averages of $p^{2}$ and $q^{2}$ as $\alpha^{2}$ and $\beta^{2}$, respectively, and set $\left(\mathbf{k}_{f} / 2-\mathbf{p}\right.$ $+\mathbf{q})^{2} \approx\left(k_{f}^{2} / 4+\alpha^{2}+\beta^{2}\right)$ in Eq. (3.3), where the average values of the scalar products are assumed to be zero. After taking an inverse Fourier transformation in Eq. (3.3) and transforming the set of variables $\mathbf{x}, \mathbf{r}_{1}, \mathbf{r}_{2}$ to $\mathbf{t}_{2}, \mathbf{r}_{1}, \mathbf{r}_{2}$, where the Jacobian is unity, the final model exchange potential becomes

$$
\begin{aligned}
g\left(\mathbf{k}_{f}, \mathbf{k}_{i}\right) \approx & -\frac{4(-1)^{l+l^{\prime}+1}}{k_{f}^{2} / 4+\alpha^{2}+\beta^{2}} \\
& \times \int \phi^{*}\left(\mathbf{r}_{2}\right) \exp \left(i \mathbf{Q} \cdot \mathbf{r}_{2}\right) \phi\left(\mathbf{r}_{2}\right) d \mathbf{r}_{2} \\
& \times \int \chi^{*}\left(\mathbf{t}_{2}\right) \exp \left(i \mathbf{Q} \cdot \mathbf{t}_{2} / 2\right) \chi\left(\mathbf{t}_{2}\right) d \mathbf{t}_{2},
\end{aligned}
$$

where $l\left(l^{\prime}\right)$ is the angular momentum of the initial (final) Ps state and Eq. (3.4) has been multiplied by $(-1)$. This provides the correct sign of the exchange potential given by formal antisymmetrization for elastic and all Ps excitation channels. This exchange potential could be considered to be a generalization of the Rudge-type exchange Born amplitude [12] for electron-impact scattering to more complex situations. For a general HF orbital (2.6), summing over appropriate target electrons $j$ and allowing for inelastic Ps channels, the (target-elastic) model exchange potential is given by

$$
\begin{aligned}
B_{E, \mu^{\prime} \mu}\left(\mathbf{k}_{f}, \mathbf{k}_{i}\right)= & \sum_{j} s_{j} g_{j} \\
= & -\left[\sum_{j} \sum_{\kappa \kappa^{\prime}} s_{j} \frac{4 a_{\kappa j} a_{\kappa^{\prime} j}(-1)^{l^{\prime}+1}}{D_{\kappa \kappa^{\prime} j}}\right. \\
& \left.\times \int \phi_{\kappa^{\prime} j}^{*}(\mathbf{r}) \exp (i \mathbf{Q} \cdot \mathbf{r}) \phi_{\kappa j}(\mathbf{r}) d \mathbf{r}\right] \\
& \times \int \chi_{n^{\prime} l^{\prime}}^{*}(\mathbf{t}) \exp (i \mathbf{Q} \cdot \mathbf{t} / 2) \chi_{n l}(\mathbf{t}) d \mathbf{t},
\end{aligned}
$$

with 


$$
D_{\kappa \kappa^{\prime} j}=\left[k_{f}^{2} / 4+\alpha_{\kappa j}^{2}+\beta_{n^{\prime}}^{2}\right],
$$

where $\mu \equiv n l\left(\mu^{\prime} \equiv n^{\prime} l^{\prime}\right)$ are the initial (final) Ps quantum numbers, $\phi_{\kappa j}(\mathbf{r})$ is the $\kappa$ th function of the $j$ th electron for the atomic ground state, and $\beta_{n^{\prime}}$ corresponds to the final inelastic Ps state, for which the derivation of the model potential is similar and leads to the same result (3.4) or (3.5). For Ps ionization, the constant $\beta_{n^{\prime}}^{2}$, which corresponds to the final Ps-state binding energy, is taken as 0 in Eq. (3.6).

As noted in Sec. II, the exchange potential given by Eqs. (3.5) and (3.6) is not time-reversal symmetric. However, if one performs in Eq. (3.1) the integration over the coordinate of the initial projectile electron $\mathbf{r}_{2}$ first with a similar average-value prescription as above, one will obtain ex- change potential (3.5) with $D_{\kappa \kappa^{\prime} j}=\left[k_{i}^{2} / 4+\alpha_{\kappa^{\prime} j}^{2}+\beta_{n}^{2}\right]$. These two possibilities suggest the following symmetric prefactor:

$$
D_{\kappa \kappa^{\prime} j}=\left[\left(k_{f}^{2}+k_{i}^{2}\right) / 8+\left(\alpha_{\kappa j}^{2}+\alpha_{\kappa^{\prime} j}^{2}\right) / 2+\left(\beta_{n^{\prime}}^{2}+\beta_{n}^{2}\right) / 2\right] .
$$

For the Ps-He system both choices (3.6) and (3.7) lead to good numerical results. At high energies the results are independent of this choice. At low energies they are sensitive to the choice and the value of the parameter $\alpha$ in Eqs. (3.6) and (3.7). In this work we shall present only results of choice (3.6) with the original and modified value of the parameter $\alpha$.

The target-elastic direct Born Ps-He amplitude for Ps transition from state $\mu$ to $\mu^{\prime}$ is given by [7]

$$
B_{D, \mu^{\prime} \mu}\left(\mathbf{k}_{f}, k_{i}\right)=\frac{4}{Q^{2}}\left[2-\sum_{\kappa \kappa^{\prime}} \sum_{j} a_{\kappa j} a_{\kappa^{\prime} j} \int \phi_{\kappa^{\prime} j}^{*}(\mathbf{r}) \exp (i \mathbf{Q} \cdot \mathbf{r}) \phi_{\kappa j}(\mathbf{r}) d \mathbf{r}\right] \int \chi_{\mu^{\prime}}^{*}(\mathbf{t})[\exp (i \mathbf{Q} \cdot \mathbf{t} / 2)-\exp (-i \mathbf{Q} \cdot \mathbf{t} / 2)] \chi_{\mu}(\mathbf{t}) d \mathbf{t}
$$

With the present prescription, the Ps-impact exchange potential is written in the form of a product of projectile and target form factors, as the direct potential above. This simple form of the amplitudes facilitates numerical calculations.

\section{B. Numerical application to Ps-He scattering}

In the case of target-elastic Ps-He scattering, electron exchange between the incident Ps and target He is only possible between like spins. Consequently, only the spin-triplet state of the electrons undergoing exchange is possible. We define appropriately symmetrized spin-triplet "Born" amplitudes, $\mathcal{B}$, via $\mathcal{B}_{\mu^{\prime} \mu}\left(\mathbf{k}_{f}, \mathbf{k}_{i}\right)=B_{D, \mu^{\prime} \mu}\left(\mathbf{k}_{f}, \mathbf{k}_{i}\right)-B_{E, \mu^{\prime} \mu}\left(\mathbf{k}_{f}, \mathbf{k}_{i}\right)$. The appropriately symmetrized scattering amplitude $f$ satisfies the following momentum-space Lippmann-Schwinger scattering integral equation [6]:

$$
\begin{aligned}
f_{\mu^{\prime} \mu}\left(\mathbf{k}^{\prime}, \mathbf{k}\right)= & \mathcal{B}_{\mu^{\prime} \mu}\left(\mathbf{k}^{\prime}, \mathbf{k}\right) \\
& -\sum_{\mu^{\prime \prime}} \int \frac{d \mathbf{k}^{\prime \prime}}{2 \pi^{2}} \frac{\mathcal{B}_{\mu^{\prime} \mu^{\prime \prime}}\left(\mathbf{k}^{\prime}, \mathbf{k}^{\prime \prime}\right) f_{\mu^{\prime \prime} \mu}\left(\mathbf{k}^{\prime \prime}, \mathbf{k}\right)}{E-\epsilon_{\mu^{\prime \prime}}-k^{\prime \prime 2} / 4+i 0},
\end{aligned}
$$

where $\epsilon_{\mu^{\prime \prime}}$ is the total energy of the Ps and He states in the intermediate state $\mu^{\prime \prime}$ and $E$ is the total energy of the system. The differential cross section is defined by $(d \sigma / d \Omega)_{\mu^{\prime}, \mu}$ $=\left(k^{\prime} / k\right)\left|f_{\mu^{\prime} \mu}\left(\mathbf{k}^{\prime}, \mathbf{k}\right)\right|^{2}$.

We performed static exchange [with $\mu^{\prime \prime}=\mathbf{P s}(1 s)$ in Eq. (3.9)] and three-Ps-state [with $\mu^{\prime \prime}=\operatorname{Ps}(1 s, 2 s, 2 p)$ in Eq. (3.9)] calculations using exact wave functions for Ps and HF atomic orbitals for He [16]. After a partial-wave projection, Eq. (3.9) was solved by the method of matrix inversion. The maximum number of partial waves included in the calculation was 10. Contribution of higher partial waves to cross sections was included by corresponding Born terms. To predict the cross sections at medium energies, we also calculated the discrete excitation $(3 s, 3 p, 3 d, 4 s, 4 p, 4 d, 4 f$,
$5 p, 5 d, 5 f$, and $6 p$ ) and ionization cross sections of Ps in the first Born approximation keeping the target frozen to its initial ground state using the present exchange model.

In Fig. 3 we plot the present target-elastic total cross section $[\operatorname{Ps}(1 s, 2 s, 2 p)$ three-Ps-state cross section plus targetelastic total Born cross sections for $n \geqslant 3$ Ps excitations and Ps ionization]. The experimental total Ps-He cross sections of two different groups-recent low-energy cross section of Ref. [2] and medium- to high-energy cross sections of Ref. [1] - are also plotted. For comparison we also plot the staticexchange and 22-coupled-pseudostate (without exchange) cross sections of Refs. [6] and [7], respectively. The measured Ps-impact total cross section of Ref. [1] has a peak near $20 \mathrm{eV}$ and a lowering trend below this energy, and the

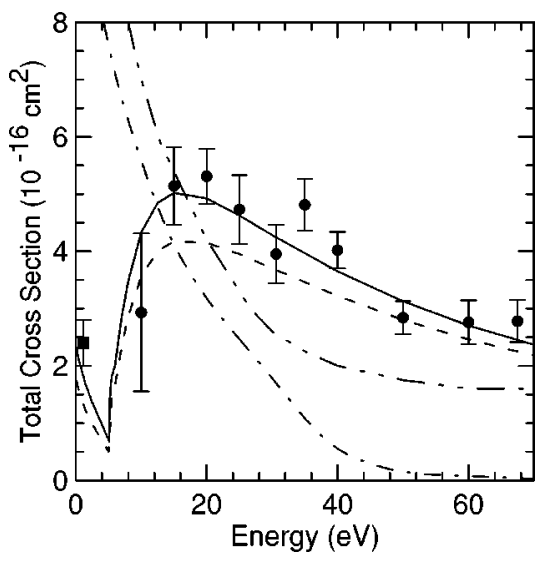

FIG. 3. Total Ps-He cross sections at different positronium energies: present target-elastic result from three-Ps-state model plus present first exchange Born for $n \geqslant 3$ excitations and ionization of Ps (dashed line); present target-elastic result with modified parameter $\alpha_{\kappa j}^{2}$ in the prefactor (full line); static-exchange model of Sarkar and Ghosh (dashed-dotted line, Ref. [6]); 22-coupled-pseudostate model of McAlinden et al. (dashed-double-dotted line, Ref. [7]); experiment (square, Ref. [2]; circle, Ref. [1]). 


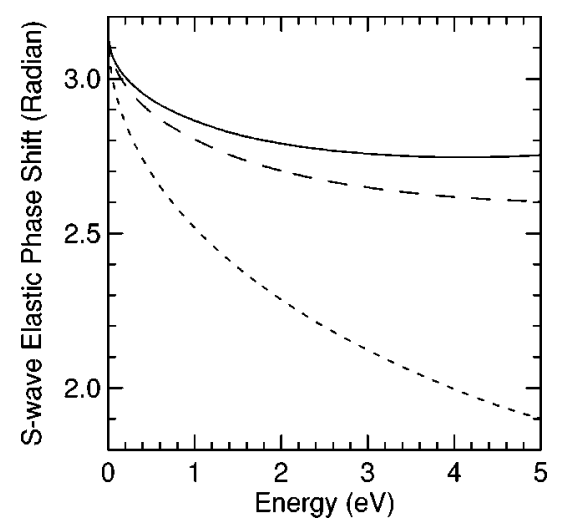

FIG. 4. $S$-wave elastic Ps-He phase shifts at different positronium energies: present three-Ps-state model (full line); present static-exchange model (dashed line); static-exchange model of Sarkar and Ghosh (dotted line, Ref. [6]).

recent measurement around $1 \mathrm{eV}$ of Ref. [2] is consistent with this trend. However, due to the large error bar of the measurement of Ref. [1] at the lowest energy $(10 \mathrm{eV})$ and due to inadequate data in this energy region, it is not clear from experiment whether the total cross section has a minimum near the Ps-excitation threshold or not. This question is addressed in the present theoretical investigation. At energies below the Ps-excitation thresholds, the elastic cross section is found to be a monotonically decreasing function of energy, as is usually found in many similar scattering problems. In the narrow energy band between 5.1 and $6.8 \mathrm{eV}$, all the Ps-inelastic channels open up causing a sharp increase of the total cross section, as can be seen in Fig. 7, resulting in a minimum of total cross section near the Ps-excitation threshold. With this feature of the cross section, the present calculation bridges the two different experimental findings and points out a minimum in total cross section near the $\operatorname{Ps}(2 s)$ threshold. This feature is also noticed in the unpublished theoretical work of Peach [19]. While the 22-coupledpseudostate calculation [7], which includes the Ps excitation and ionization effects through pseudostates, completely denies this trend; the static-exchange cross sections [4-6] are too large to match the measurement near $\operatorname{Ps}(2 s)$ threshold.

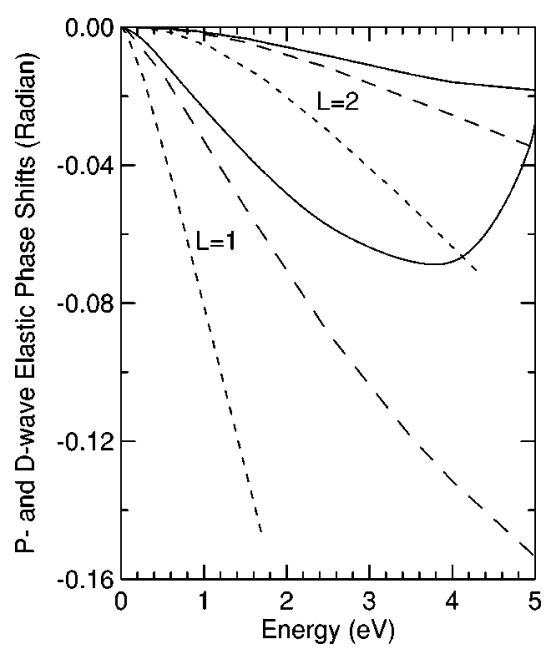

FIG. 5. $P$ - and $D$-wave elastic Ps-He phase shifts at different positronium energies: notations are the same as in Fig. 4.

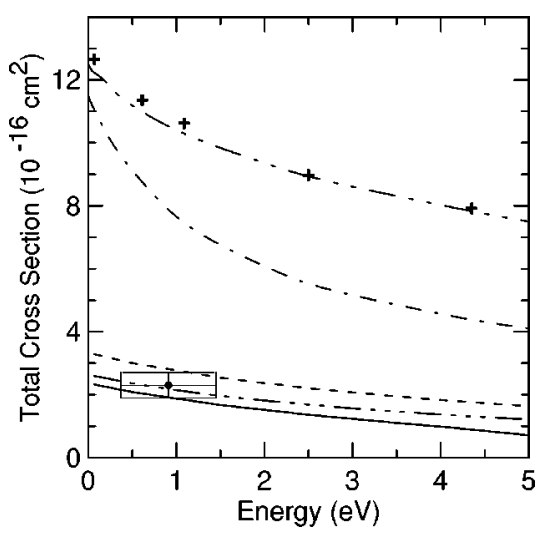

FIG. 6. Angle-integrated Ps-He elastic cross section at low positronium energies: present three-Ps-state model (full line); present static exchange model (dotted line); present static exchange with unmodified parameter $\alpha$ (dashed-tripple-dotted line); Fraser (dashed-double-dotted line, Ref. [4]); Barker and Bransden (dashed-dotted line, Ref. [5]); Sarkar and Ghosh (plus, Ref. [6]); experiment shown by the rectangle (Ref. [2]).

So far we have parametrized the model potential from a physical argument and presented results with it. In Eq. (3.5), $\alpha_{\kappa j}$ 's are parameters of HF orbitals. In Fig. 3 we also exhibit the consequence of a small variation of $\alpha_{\kappa j}$ in the prefactor $\left(k_{f}^{2} / 4+\alpha_{\kappa j}^{2}+\beta_{n^{\prime}}^{2}\right)^{-1}$ of Eq. (3.5). The full line, providing an overall better agreement with experiment, is obtained by varying parameters $\alpha_{\kappa j}$ 's in the prefactor (3.6), which is taken as

$$
D_{\kappa \kappa^{\prime} j}=\left[k_{f}^{2} / 4+\left(0.88 \alpha_{\kappa j}\right)^{2}+\beta_{n^{\prime}}^{2}\right]
$$

in both the static-exchange and three-Ps-state calculations. Unless specifically mentioned, all results presented here are calculated with this modified prefactor. The above reduction in the average value of $\left\langle p^{2}\right\rangle$ has led to a better agreement with experiment.

Next we present an account of phase shifts and angleintegrated partial cross sections with modified prefactor (3.10). The present static-exchange and three-Ps-state elastic

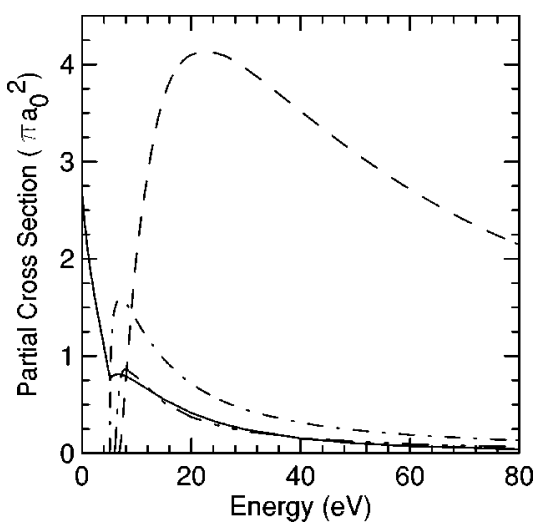

FIG. 7. Angle-integrated Ps-He partial cross sections at different positronium energies with exact $\alpha$ : present elastic from three-Psstate model (full line) and $\operatorname{Ps}(2 s+2 p)$ excitation (dashed-dotted line) from three-Ps-state model, present $\operatorname{Ps}(n \geqslant 3)$ excitation (dashed-double-dotted line), and Ps ionization (dashed line) using first Born approximation with present exchange. 
TABLE I. Angle-integrated Ps-He partial cross sections in $\pi a_{0}^{2}$ at different positronium energies: EB, first Born with present exchange; PSE, present static exchange; SE, static exchange of Ref. [6]; TPS, three-Ps-state with present exchange.

\begin{tabular}{|c|c|c|c|c|c|c|c|c|c|c|}
\hline $\begin{array}{l}\text { Energy } \\
(\mathrm{eV})\end{array}$ & $\begin{array}{c}\operatorname{Ps}(1 s) \\
\text { EB }\end{array}$ & $\begin{array}{c}\operatorname{Ps}(2 s) \\
\mathrm{EB}\end{array}$ & $\begin{array}{c}\operatorname{Ps}(2 p) \\
\mathrm{EB}\end{array}$ & $\begin{array}{c}\operatorname{Ps}(1 s) \\
\text { SE }\end{array}$ & $\begin{array}{c}\operatorname{Ps}(1 s) \\
\text { PSE }\end{array}$ & $\begin{array}{c}\operatorname{Ps}(1 s) \\
\text { TPS }\end{array}$ & $\begin{array}{c}\operatorname{Ps}(2 s) \\
\text { TPS }\end{array}$ & $\begin{array}{c}\operatorname{Ps}(2 p) \\
\text { TPS }\end{array}$ & $\begin{array}{c}\operatorname{Ps}(n \geqslant 3) \\
\mathrm{EB}\end{array}$ & $\begin{array}{c}\text { Ps ion } \\
\text { EB }\end{array}$ \\
\hline 0.068 & 13.73 & & & 14.4 & 3.73 & 2.70 & & & & \\
\hline 0.612 & 10.88 & & & 12.9 & 3.34 & 2.36 & & & & \\
\hline 1.088 & 9.05 & & & 12.1 & 3.07 & 2.13 & & & & \\
\hline 1.7 & 7.31 & & & 11.3 & 2.80 & 1.88 & & & & \\
\hline 2.448 & 5.79 & & & 10.5 & 2.52 & 1.62 & & & & \\
\hline 4.352 & 3.57 & & & 9.0 & 1.99 & 1.09 & & & & \\
\hline 5 & 3.10 & & & & 1.85 & 0.89 & & & & \\
\hline 5.508 & 2.81 & $0.80(-1)$ & 1.51 & & 1.75 & 0.81 & $0.49(-1)$ & 0.83 & & \\
\hline 6 & 2.56 & 0.10 & 1.87 & & 1.66 & 0.81 & $0.70(-1)$ & 1.16 & & \\
\hline 6.8 & 2.22 & 0.12 & 1.98 & 7.7 & 1.53 & 0.81 & $0.74(-1)$ & 1.39 & 0.69 & \\
\hline 8 & 1.84 & 0.11 & 1.86 & & 1.35 & 0.79 & $0.64(-1)$ & 1.44 & 0.86 & 0.74 \\
\hline 10 & 1.39 & $0.91(-1)$ & 1.54 & & 1.11 & 0.73 & $0.52(-1)$ & 1.31 & 0.78 & 2.05 \\
\hline 15 & 0.80 & $0.54(-1)$ & 1.00 & & 0.71 & 0.55 & $0.45(-1)$ & 0.94 & 0.52 & 3.67 \\
\hline 20 & 0.52 & $0.35(-1)$ & 0.72 & 3.6 & 0.49 & 0.41 & $0.33(-1)$ & 0.68 & 0.38 & 4.10 \\
\hline 30 & 0.27 & $0.17(-1)$ & 0.44 & 2.0 & 0.26 & 0.24 & $0.18(-1)$ & 0.43 & 0.23 & 3.96 \\
\hline 40 & 0.16 & $0.10(-1)$ & 0.31 & 0.6 & 0.16 & 0.15 & $0.11(-1)$ & 0.30 & 0.16 & 3.52 \\
\hline 50 & 0.11 & $0.65(-2)$ & 0.23 & & 0.11 & 0.10 & $0.68(-2)$ & 0.23 & 0.11 & 3.09 \\
\hline 60 & $0.75(-1)$ & $0.45(-2)$ & 0.19 & $0.8(-1)$ & $0.75(-1)$ & $0.72(-1)$ & $0.46(-2)$ & 0.18 & $0.93(-1)$ & 2.72 \\
\hline 80 & $0.41(-1)$ & $0.24(-2)$ & 0.13 & $0.1(-1)$ & $0.41(-1)$ & $0.40(-1)$ & $0.25(-2)$ & 0.13 & $0.63(-1)$ & 2.14 \\
\hline 100 & $0.25(-1)$ & $0.14(-2)$ & $0.98(-1)$ & $0.2(-2)$ & $0.25(-1)$ & $0.25(-1)$ & $0.14(-2)$ & $0.98(-1)$ & $0.49(-1)$ & 1.74 \\
\hline
\end{tabular}

scattering phase shifts for different partial waves below the lowest excitation threshold are shown in Figs. 4 ( $S$ wave) and 5 ( $P$ and $D$ waves). The present phase shifts are different from those of previous calculations [4-6], as is expected from the cross-section pattern. However, for comparison we show the phase shifts of the recent work by Sarkar and Ghosh [6] in Figs. 4 and 5. At these energies the S-wave phase shifts alone control the elastic cross section. The present low-energy elastic S-wave phase shifts are expected to be more plausible to those of the previous calculations as from Fig. 3 we find that the present cross sections are in better agreement with experiment.

In Fig. 6 we plot the present low-energy elastic cross sections for static-exchange and three-Ps-state calculations. We compare these cross sections with the recent sophisticated low-energy experimental cross section of Ref. [2] measured using time-resolved Doppler broadening spectroscopy and the previous static-exchange cross sections of Refs. [4-6]. Here, for experimental purposes, we also plot the present static-exchange cross section calculated with the exact parameter $\alpha$ in the prefactor. The present three-Ps-state cross sections are significantly smaller than previous theoretical cross sections and are in close agreement with experiment. The present exchange Born cross sections are also smaller than those of previous calculations. For example, Sarkar and Ghosh [6] obtained the first Born elastic cross sections $131.9 \pi a_{0}^{2}$ and $12.5 \pi a_{0}^{2}$ at $0.068 \mathrm{eV}$ and $1 \mathrm{eV}$, compared to the present first Born elastic cross sections $13.73 \pi a_{0}^{2}$ and $9.0 \pi a_{0}^{2}$, respectively. This implies that the resulting repulsive potential of the present model is much weaker compared to previous ones. This, in turn, would allow the Ps to be closer to the He atom, which is expected to lead to a higher value of $Z_{\text {eff }}$ as required to satisfy the ex- periment [5]. The present three-Ps-state cross sections are smaller than the static-exchange cross sections and are well within the experimental error bar.

In Fig. 7 we exhibit the different angle-integrated partial cross sections for the three-Ps-state calculation. Here we show the $\operatorname{Ps}(1 s)$ and $\operatorname{Ps}(2 s+2 p)$ cross sections from the three-Ps-state calculation and $\operatorname{Ps}(n \geqslant 3)$ excitation and Psionization cross sections using the present total first Born model. At medium energies the Ps-ionization cross sections are the largest and dominate the trend of the total cross section of Fig. 3. This feature has also been observed by Campbell et al. [7] in Ps-H scattering.

The angle-integrated partial cross sections are of crucial importance and are presented in Table I. These partial cross sections are calculated with the modified prefactor (3.10) and leads to total cross sections in better agreement with experiment. These cross sections should be considered to be the most realistic results of the present model study except near zero energy, where van der Waals force might play a crucial role, which is not taken into account. In addition to the threePs-state cross sections, we also present our first Born and static-exchange results in Table I with modified prefactor (3.10). For comparison we also show the static-exchange results of Sarkar and Ghosh [6]. The present elastic Born cross sections are much smaller than those of Ref. [6]. The present three-Ps-state elastic cross sections are smaller than the present static-exchange cross sections, which demonstrates the effect of large polarizability of Ps. The present exchange Born (EB) results are in close agreement with three-Ps-state results for energies greater than $20 \mathrm{eV}$ (see Table I). So present EB cross sections for Ps $(n \geqslant 3)$ excitations and ionization are expected to be close to converged ones beyond 20 $\mathrm{eV}$. The nonconvergence of these cross sections near 6-15 
$\mathrm{eV}$ is responsible for the overestimation of the total cross section at these energies (see Fig. 3).

\section{SUMMARY}

We have presented simple model exchange potentials for electron- and Ps-impact scattering suitable for performing dynamical calculation in many-electron systems and checked it in electron scattering from $\mathrm{H}$ and $\mathrm{He}$ and applied it to Ps scattering from He. The present static-exchange and threestate coupled-channel cross sections of electron-impact scattering are in agreement with other existing results $[10,14,15,17,18]$. We also have performed static-exchange and three-Ps-state calculations for Ps-He scattering at low and medium energies. To exhibit the usefulness of the present exchange at medium energies, higher excitations and ionization of Ps are calculated using the first Born model with present exchange. The present target-elastic total cross sections agree well with experiment $[1,2]$ both at low and medium energies.

\section{ACKNOWLEDGMENTS}

The work was supported in part by the Conselho Nacional de Desenvolvimento Científico e Tecnológico and Fundação de Amparo à Pesquisa do Estado de São Paulo of Brazil.
[1] A. J. Garner, G. Laricchia, and A. Ozen, J. Phys. B 29, 5961 (1996).

[2] M. Skalsey, J. J. Engbrecht, R. K. Bithell, R. S. Vallery, and D. W. Gidley, Phys. Rev. Lett. 80, 3727 (1998).

[3] P. K. Biswas and S. K. Adhikari, J. Phys. B 31, 3147 (1998), and references therein.

[4] P. A. Fraser, J. Phys. B 1, 1006 (1968).

[5] M. I. Barker and B. H. Bransden, J. Phys. B 1, 1109 (1968).

[6] N. K. Sarkar and A. S. Ghosh, J. Phys. B 30, 4591 (1997).

[7] M. T. McAlinden, F. G. R. S. MacDonald, and H. R. J. Walters, Can. J. Phys. 74, 434 (1996).

[8] P. K. Biswas and S. K. Adhikari, J. Phys. B 31, L315 (1998).

[9] M. J. Seaton, Philos. Trans. R. Soc. London, Ser. A 245, 469 (1953); P. G. Burke and M. J. Seaton, Methods Comput. Phys. 10, 1 (1971); A. T. Stelbovics and B. H. Bransden, J. Phys. B 22, L451 (1989).

[10] P. G. Burke and H. M. Schey, Phys. Rev. 126, 147 (1962); A. Temkin and J. C. Lamkin, ibid. 121, 788 (1961).

[11] M. E. Riley and D. G. Truhlar, J. Chem. Phys. 63, 2182 (1975); A. W. Fliflet and V. McKoy, Phys. Rev. A 21, 1863 (1980); F. A. Gianturco, XIX ICPEAC, edited by L. J. Dubé et al., AIP Conf. Proc. No. 360 (AIP Press, Woodbury, 1995), p. 211; S. Hara, J. Phys. Soc. Jpn. 22, 710 (1967); M. A. Morrison, A. N. Feldt, and D. Austin, Phys. Rev. A 29, 2518 (1984); P. Baille and J. W. Darewych, J. Phys. B 10, L615 (1977).

[12] M. R. H. Rudge, Proc. Phys. Soc. London 86, 763 (1965); V. I. Ochkur, Zh. Eksp. Teor. Fiz. 45, 734 (1963) [Sov. Phys. JETP 18, 503 (1964)].

[13] J. R. Oppenheimer, Phys. Rev. 32, 361 (1928).

[14] R. H. Neynaber, L. L. Marino, E. W. Rothe, and S. M. Trujillo, Phys. Rev. 124, 135 (1961).

[15] J. Callaway, Phys. Rev. A 32, 775 (1985).

[16] E. Clementi and C. Roetti, At. Data Nucl. Data Tables 14, 177 (1974).

[17] D. Andrick and H. Bitsch, J. Phys. B 8, 393 (1975); F. J. de Heer and R. H. J. Jansen, ibid. 10, 3741 (1977); D. F. Register, S. Trajmar, and S. K. Srivastava, Phys. Rev. A 21, 1134 (1980); D. E. Golden and H. W. Bandel, Phys. Rev. 138, A14 (1965).

[18] P. G. Burke, J. W. Cooper, and S. Ormonde, Phys. Rev. 183, 245 (1969).

[19] G. Peach (unpublished), as quoted in Ref. [1]. 